\title{
MicroRNA-7 modulates cellular senescence to relieve gemcitabine resistance by targeting PARP1/NF-кB signaling in pancreatic cancer cells
}

\author{
ZHI-QIANG YE ${ }^{1}$, HAN-BIN CHEN ${ }^{2}$, TAI-YU ZHANG ${ }^{3}$, ZHI CHEN $^{4}$, LING TIAN $^{5}$ and DIAN-NA GU ${ }^{2}$
}

Departments of ${ }^{1}$ Thyroid and Breast Surgery, and ${ }^{2}$ Chemoradiotherapy, The First Affiliated Hospital of Wenzhou Medical University, Wenzhou, Zhejiang 325000; ${ }^{3}$ Institute of Translational Medicine, Shanghai General Hospital,

Shanghai Jiao Tong University School of Medicine, Shanghai 201620; ${ }^{4}$ Department of Chemoradiotherapy, The Second Affiliated Hospital of Wenzhou Medical University, Wenzhou, Zhejiang 325000; ${ }^{5}$ Department of Central Laboratory, Shanghai Chest Hospital, Shanghai Jiao Tong University School of Medicine, Shanghai 200030, P.R. China

Received May 11, 2020; Accepted November 11, 2020

DOI: $10.3892 / 01.2020 .12400$

\begin{abstract}
Senescence is activated in response to gemcitabine to prevent the propagation of cancer cells. However, there is little evidence on whether senescence is involved in gemcitabine resistance in pancreatic cancer. Increasing evidence has demonstrated that microRNAs (miRs) are potential regulators of cellular senescence. The present study aimed to investigate whether aberrant miR-7 expression modulated senescence to influence pancreatic cancer resistance to chemotherapy. In the present study, cell senescence assay, ALDEFLUOR ${ }^{\mathrm{TM}}$ assay, luciferase reporter assay, flow cytometry, quantitative PCR, immunohistochemistry and western blot analysis were performed to explore the association between senescence and gemcitabine therapy response, and to clarify the underlying mechanisms. The present study revealed that gemcitabine-induced chronically existing senescent pancreatic cells possessed stemness markers. Therapy-induced senescence led to gemcitabine resistance. Additionally, it was found that miR-7 expression was decreased in gemcitabine-resistant pancreatic cancer cells, and that miR-7 acted as an important regulator of cellular senescence by targeting poly (ADP-ribose) polymerase 1 (PARP1)/NF- $\mathrm{BB}$ signaling. When miR-7 expression was restored, it was able to sensitize pancreatic cancer cells to gemcitabine. In conclusion,
\end{abstract}

Correspondence to: Dr Ling Tian, Department of Central Laboratory, Shanghai Chest Hospital, Shanghai Jiao Tong University School of Medicine, 241 West Huaihai Road, Shanghai 200030, P.R. China

E-mail:TL09168@hotmail.com

Dr Dian-Na Gu, Department of Chemoradiotherapy, The First Affiliated Hospital of Wenzhou Medical University, 2 Fuxue Road, Wenzhou, Zhejiang 325000, P.R. China

E-mail: yinuo801@126.com

Key words: microRNA-7, senescence, gemcitabine resistance, pancreatic cancer the present study demonstrated that miR-7 regulated cellular senescence and relieved gemcitabine resistance by targeting the PARP1/NF- $\mathrm{BB}$ axis in pancreatic cancer cells.

\section{Introduction}

Pancreatic ductal adenocarcinoma (PDAC) is the most aggressive primary pancreatic neoplasm and has the poorest prognosis among solid tumors, with a 5-year-survival rate of only $9 \%$ (1). The management of pancreatic cancer is notably difficult due to a poor response to available therapeutic modalities, such as chemotherapy and radiotherapy (2). Over the past decade, gemcitabine has been demonstrated to improve median survival time and quality of life in patients with advanced pancreatic cancer, but the prognosis of the disease remains dismal (3). Gemcitabine resistance is one of the main challenges, and the underlying mechanisms remain unclear.

It is known that numerous anticancer therapeutic agents, including doxorubicin, cisplatin and camptotecin, typically rely on causing DNA damage, which engages potent DNA damage response signaling pathways that culminate in apoptosis or growth arrest at checkpoints to allow for damage repair (4-6). Cellular senescence, as a stress-responsive cell cycle arrest program, is commonly considered as a tumor-suppressing response (7) and has been demonstrated to contribute to the outcomes of anticancer chemotherapy in vivo, such as in lymphoma (8). However, previous studies have suggested that senescence may protect cancer cells from genotoxic treatment due to a chemoprotective environment $(9,10)$. In addition, the senescence phenotype of pancreatic cancer cells induced by gemcitabine, which is characterized by enhanced senescence-associated $\beta$-galactosidase (SA- $\beta$-Gal) and increased expression levels of senescence-associated secretory phenotype (SASP), is associated with resistance to gemcitabine $(11,12)$. Thus, there is contrasting evidence on the tumor-suppressing and tumor-promoting functions of therapy-induced senescence (TIS). Therefore, the role of senescence in the chemotherapy of pancreatic cancer and the underlying mechanisms remain unclear. 
Emerging evidence has revealed that microRNAs (miRNAs/miRs) are potential regulators of cellular senescence by targeting genes involved in the production of reactive oxygen species, shortening of telomeres, mitochondrial damage and cell cycle arrest via production of tumor suppressor proteins (13). miR-7, which acts as a tumor suppressor in various gastrointestinal types of cancer, including pancreatic cancer (14), can downregulate poly (ADP-ribose) polymerase 1 (PARP1), which functions as a regulator of diverse biological processes, including DNA repair and chromatin remodeling in the senescence program $(15,16)$. Thus, considering the role of cellular senescence as a contributing factor to therapy resistance, the present study aimed to investigate whether aberrant miR-7 expression modulated senescence to influence cancer response to chemotherapy.

\section{Materials and methods}

Cell culture. The human pancreatic ductal adenocarcinoma PANC-1 cell line was obtained from The Cell Bank of Type Culture Collection of the Chinese Academy of Sciences. PANC-1 cells were cultured in DMEM supplemented with $10 \%$ FBS (both Gibco; Thermo Fisher Scientific, Inc.) at $37^{\circ} \mathrm{C}$ in a $5 \% \mathrm{CO}_{2}$ incubator. A gemcitabine-resistant pancreatic cancer cell line was established by treating PANC-1 cells with $0.25 \mu \mathrm{M}$ gemcitabine for 4 weeks.

Constructs, oligonucleotides and reagents. TargetScanHuman 7.2 (http://www.targetscan.org) was used to identify potential target genes of miR-7. To confirm the molecular interaction between miR-7 and PARP1, the total RNA of human embryonic kidney 293T cells (purchased from The Cell Bank of Type Culture Collection of the Chinese Academy of Sciences and cultured in DMEM with 10\% FBS) was exacted using TRIzol $^{\circledast}$ reagent (Invitrogen; Thermo Fisher Scientific, Inc.) and then reverse transcribed into cDNA using PrimeScript ${ }^{\mathrm{TM}}$ RT Master Mix according to standard procedures (Takara Biotechnology Co., Ltd.). PCR amplification was performed using PrimerSTAR HS DNA polymerase (Takara Biotechnology Co., Ltd.) with initial denaturation at $98^{\circ} \mathrm{C}$ for $10 \mathrm{sec}$, followed by 30 cycles of $5 \mathrm{sec}$ denaturation at $55^{\circ} \mathrm{C}$ and $1 \mathrm{~min}$ extension at $72^{\circ} \mathrm{C}$, and final extension at $72^{\circ} \mathrm{C}$ for $2 \mathrm{~min}$. The products were collected by $1 \%$ agarose gel electrophoresis using Gel-red for visualization. The 3'-untranslated region (UTR; 769 bp) of PARP1 was amplified by PCR using the following primers: forward, 5'-ACTGCTAGCGGTAATTGG GAGAGGTAGC-3' and reverse, 5'-TGAGTCGACTAGAGA AGGCATCTGCATTTTTAATC-3'. The amplified 3'-UTR was sequenced by Sangon Biotech Co., Ltd., double-digested with NheI/SalI and inserted into the corresponding digested reporter plasmid pmirGLO (7,350 bp; Promega Corporation). Similarly, the putative counterparts [wild-type (WT) and mutant (MT)] of the miR-7-target sequence in the 3'-UTR of PARP1 (GenBank NM_001618.4) were also used to construct the corresponding reporter plasmids, pmirGLO-PARP1-WT and pmirGLO-PARP1-MT, respectively. Sequencing of the luciferase reporter constructs was performed by Sangon Biotech Co.,Ltd. Synthetic miR-7-5p mimics (50 nM), miR-7-5p inhibitor $(100 \mathrm{nM})$ and their negative control oligonucleotides (non-targeting), purchased from Guangzhou RiboBio Co., Ltd., were transfected into parent and gemcitabine-resistant PANC-1 cells using Lipofectamine ${ }^{\circledR} 3000$ (Invitrogen; Thermo Fisher Scientific, Inc.) for $15 \mathrm{~min}$ at room temperature. Subsequent experiments were performed $24 \mathrm{~h}$ after transfection. The PARP signaling inhibitor rucaparib (AG-014699) phosphate was purchased from Selleck Chemicals. All the enzymes for molecular cloning were purchased from New England Biolabs, Inc. The sequences of the synthesized oligonucleotides used in the present study are listed in Table SI.

Luciferase reporter assay. To determine the miR-7 target genes, PANC-1 cells cultured in 24-well plates were co-transfected with reporter plasmids and miR-7 mimics using Lipofectamine ${ }^{\circledR} 2000$ (Invitrogen; Thermo Fisher Scientific, Inc.). Cells were harvested and lysed $48 \mathrm{~h}$ after transfection. Luciferase assays were performed using the Dual-Luciferase ${ }^{\circledR}$ Reporter Assay (cat. no. E1910; Promega Corporation) according to the manufacturer's protocol. Firefly luciferase activity was normalized to Renilla luciferase activity, and the relative luciferase score was calculated.

$R N A$ extraction and reverse transcription-quantitative $P C R$ $(R T-q P C R)$. Total RNA from PANC-1 cells was extracted using TRIzol ${ }^{\circledR}$ reagent (Invitrogen; Thermo Fisher Scientific, Inc.) and $1 \mu \mathrm{g}$ RNA was reverse transcribed into cDNA using PrimeScript ${ }^{\mathrm{TM}}$ RT reagent kit according to the manufacturer's protocol (Takara Biotechnology Co., Ltd.). qPCR was performed in triplicate with the use of the SYBR ${ }^{\circledR}$ Premix Ex Taq $^{\mathrm{TM}}$ (Takara Biotechnology Co., Ltd.) on the QuantStudio ${ }^{\mathrm{TM}}$ 6 Flex System (Applied Biosystems; Thermo Fisher Scientific, Inc.). The primers for the genes used in present study were synthesized by Sangon Biotech Co., Ltd. The thermocycling conditions were as follows: Initial denaturation at $95^{\circ} \mathrm{C}$ for $30 \mathrm{sec}$, followed by 40 cycles of $5 \mathrm{sec}$ denaturation at $95^{\circ} \mathrm{C}$ and $34 \mathrm{sec}$ extension at $60^{\circ} \mathrm{C}$. U6 small nuclear RNA and GAPDH were used as internal controls for miRNA and mRNA assays, respectively. The $2^{-\Delta \Delta C q}$ method (17) was used to calculate the relative mRNA expression level after normalization to U6 and GAPDH RNA. All the RT-qPCR primer sequences are listed in Table SII.

Western blotting. RIPA lysis buffer (Thermo Fisher Scientific, Inc.) was used to lyse and extract total protein from PANC-1 gemcitabine-resistant and parent cell lines. Protein concentration was determined used a BCA protein assay and protein lysates $(25 \mathrm{ng} / \mu \mathrm{l} ; 6 \mu \mathrm{l} / \mathrm{lane})$ were separated via 10 or $12 \%$ SDS-PAGE according to molecular weight. Subsequently, separated protein bands were transferred onto polyvinylidene fluoride membranes (PVDF) (EMD Millipore) and blocked with $5 \%$ skimmed milk for $1 \mathrm{~h}$ at room temperature. Next, the membranes were first probed with the relevant primary antibodies overnight at $4^{\circ} \mathrm{C}$ and subsequently incubated with HRP-labeled secondary antibodies for $2 \mathrm{~h}$ at room temperature for visualization using Immobilon ${ }^{\mathrm{TM}}$ Western HRP Substrate kit (EMD Millipore). The antibodies used are listed in Table SIII.

Immunofluorescence. Cells were plated onto glass coverslips, fixed with $4 \%$ paraformaldehyde for $20 \mathrm{~min}$ and permeabilized with $0.1 \%$ Triton X-100 in phosphate-buffered saline (PBS) 
for $15 \mathrm{~min}$ at room temperature. Next, 5\% BSA (Invitrogen; Thermo Fisher Scientific, Inc.) was applied for $1 \mathrm{~h}$ at room temperature, and then rabbit anti-human phospho-NF- $\kappa \mathrm{B}$ p65 (Ser536; Table SIII) was added and incubated at $4^{\circ} \mathrm{C}$ overnight. Next, Alexa Fluor 594-conjugated goat anti-rabbit secondary antibody was added and incubated for $2 \mathrm{~h}$ at room temperature. Finally, the cells were stained with DAPI to stain cell nuclei for $5 \mathrm{~min}$ at room temperature. Immunostaining signals and DAPI-stained nuclei were visualized using a confocal microscope at x40 magnification (TCS SP8; Leica Microsystems, Inc.).

ALDEFLUOR ${ }^{T M}$ assay. The ALDEFLUOR ${ }^{\mathrm{TM}}$ kit (Stemcell Technologies, Inc.) was used to analyze the population with aldehyde dehydrogenase (ALDH) enzymatic activity according to the manufacturer's protocol. Briefly, cells were incubated in the ALDEFLUOR ${ }^{\mathrm{TM}}$ assay buffer containing the $\mathrm{ALDH}$ substrate BAAA at $37^{\circ} \mathrm{C}$ for $45 \mathrm{~min}$, whereas control cells were incubated with $50 \mathrm{mM}$ ALDH inhibitor diethylamino-benzaldehyde under the same conditions. BAAA-stained cells were analyzed using a FACSCalibur flow cytometer (Becton, Dickinson and Company) with CellQuest software (version 4.0.2; Becton, Dickinson and Company).

Flow cytometry assay. The ratio of $\mathrm{CD} 24^{+} \mathrm{CD} 326^{+}$subpopulation was evaluated using flow cytometry assay. Briefly, the cells were collected, resuspended in binding buffer and labeled with $10 \mu \mathrm{l}$ PE mouse anti-human CD24 antibody and $10 \mu \mathrm{l}$ BB515 mouse anti-human CD326 antibody (Table SIII) in the dark at room temperature for $15 \mathrm{~min}$. Cells were then analyzed using a FACSCalibur flow cytometer (Becton, Dickinson and Company) with CellQuest software (version 4.0.2; Becton, Dickinson and Company) to determine the percentage of CD $24^{+}$CD $326^{+}$cells.

Tumorsphere culture. The tumorsphere culture method and medium preparation were performed according to a previous study (18). PANC-1 cells were plated in 6-well ultralow attachment plates (Corning Inc.) at a concentration of 10,000 cells/well. After 14 days, spheres with $\geq 50 \mu \mathrm{m}$ in diameter were counted under an inverted phase-contrast light microscope at x10 magnification.

Cell viability assay. Cells were plated at a density of $2 \times 10^{5} /$ well in 6-well plates with different concentrations $(0,0.1,0.25$ and $1.0 \mu \mathrm{M}$ ) of gemcitabine at $37^{\circ} \mathrm{C}$ in a $5 \% \mathrm{CO}_{2}$ incubator for $24 \mathrm{~h}$. After 2 days, cells were collected and counted using a hemocytometer.

Cell senescence assay. Cell senescence was detected using a SA- $\beta$-Gal assay kit (Cell Signaling Technology, Inc.), following the manufacturer's protocol. The cells with different treatments were grown on 6-well culture plates, washed and fixed for $15 \mathrm{~min}$ at room temperature with $4 \%$ paraformaldehyde. Subsequently, cells were washed with PBS three times and incubated with $\beta$-galactosidase staining solution overnight at $37^{\circ} \mathrm{C}(\mathrm{pH} 6.0)$. A light microscope at x10 magnification was used to collect the image files and the percentage of blue cells was calculated using Image-Pro Plus software (version 6.0; Media Cybernetics, Inc.).
EdU assay. Cell proliferation was analyzed using a Cell-Light EdU DNA Cell Proliferation kit (Guangzhou RiboBio Co., Ltd.) according to the manufacturer's protocol. PANC-1 parent and gemcitabine-resistant cells were detected using a fluorescence microscope (magnification, x20), and cell proliferation was determined as the ratio of EdU-positive cells.

Cell Counting Kit-8 (CCK-8) assay. The CCK-8 assay (Dojindo Molecular Technologies, Inc.) was performed to assess gemcitabine-resistant PANC-1 cell viability according to the manufacturer's protocol. Briefly, cells were incubated in 96-well plates with $10 \mu \mathrm{lCCK}-8$ reagent per well for $2 \mathrm{~h}$ at $37^{\circ} \mathrm{C}$. Subsequently, cell viability was measured at a wavelength of $450 \mathrm{~nm}$ using a microplate reader.

Tissue microarrays. The pancreatic tissue microarray HPan-Ade180Sur-02 was purchased from Shanghai Xinchao Biological Technology Co., Ltd. HPan-Ade180Sur-02 incorporated 100 cases of pancreatic tumor and 80 cases of adjacent non-tumor tissues, of which 63 males and 37 females. The median age was 62 years (range, $34-85$ years). All the raw data including overall survival are available from Shanghai Xinchao Biological Technology Co., Ltd.

Immunohistochemistry. Immunohistochemistry was performed on tissue microarray chips (Shanghai Xinchao Biological Technology Co., Ltd.). Paraffin-embedded sections were immersed in xylene solution to dewax for $10 \mathrm{~min}$ twice at room temperature and rehydrated in 100, 95, 85 and $75 \%$ gradient ethanol. Subsequently, $3 \%$ hydrogen peroxide was used for quenching. After recovering the antigens and blocking with 5\% BSA (Invitrogen; Thermo Fisher Scientific, Inc.) at $37^{\circ} \mathrm{C}$ for $1 \mathrm{~h}$, the slides were probed with the primary antibody rabbit anti-human histone $\mathrm{H} 3$ (trimethyl $\mathrm{K} 9$ ) at $4^{\circ} \mathrm{C}$ overnight and then with an HRP-conjugated goat anti-rabbit secondary antibody for $2 \mathrm{~h}$ at room temperature (Table SIII). The proteins were visualized in situ with DAB chromogenic substrate. The sections were observed using light microscopy at a magnification of $\mathrm{x} 20$. The results of immunostaining were evaluated as follows: The mean percentage of stained tumor cells per specimen was determined semi-quantitatively and scored as 0 for no positive cells, 1 for $<10 \%$ positive cells, 2 for $10-50 \%$ positive cells and 3 for $>50 \%$ positive cells. The intensity of staining was determined as 0 for no staining, 1 for weak staining, 2 for moderate staining and 3 for strong staining. The staining index (SI) for each specimen was calculated as the product of the staining intensity by the percentage of positive tumor cells. A SI of 0-3 represented negative triMeH3K9 expression, while a SI of 4-9 represented positive triMeH3K9 expression.

Bioinformatic analysis. Raw RNA-seq data and survival data for 175 pancreatic tumors were downloaded from The Cancer Genome Atlas (TCGA) pancreatic ductal adenocarcinoma project (http://oncolnc.org). Data were analyzed for cyclin-dependent kinase inhibitor 1A (CDKN1A/p21) and Ki67 gene expression. The expression levels of CDKN1A were listed from the lowest to the highest. The corresponding patients were numbered 1 to 175 in sequence. The expression levels of Ki67 were listed from the highest to the lowest, and 
A

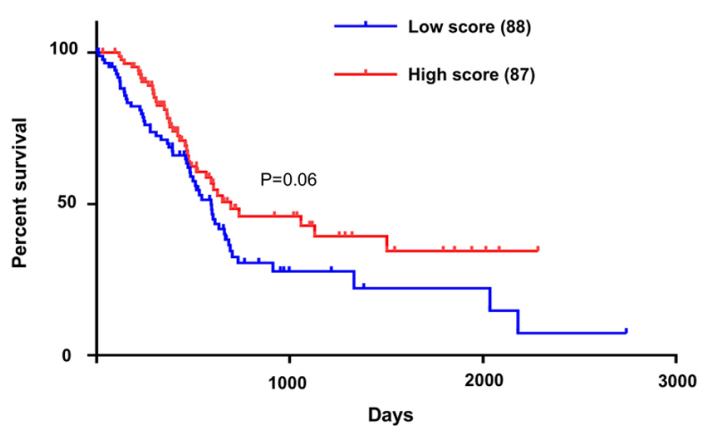

C

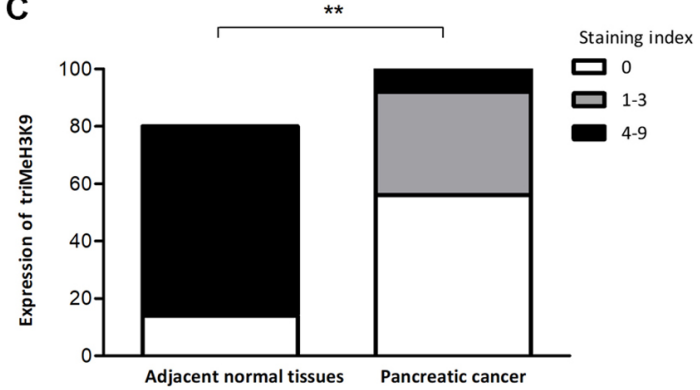

B

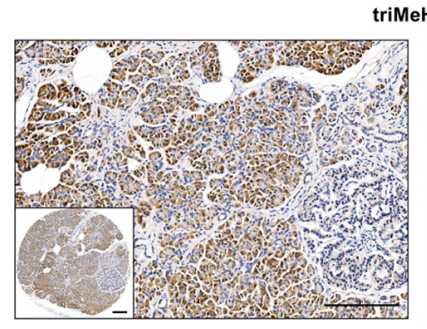

Adjacent normal (staining index :9)

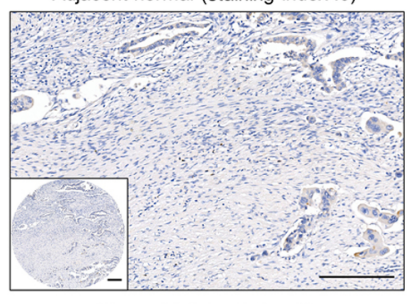

Tumor (staining index :0) survival time 58 months

D

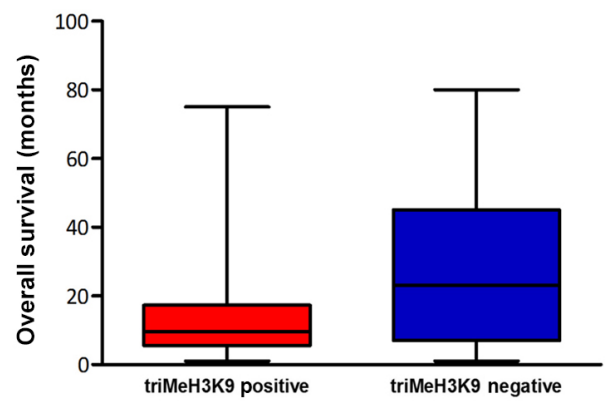

Figure 1. Association between cellular senescence and prognosis in patients with pancreatic cancer. (A) Kaplan-Meier analysis was performed to classify the 175 patients with pancreatic cancer according to the senescent score, which was calculated based on CDKN1A/p21 positivity in combination with Ki67 negativity in pancreatic cancer from The Cancer Genome Atlas dataset. (B) Immunohistochemical analysis of pancreatic tissue microarrays using an antibody against the senescent marker triMeH3K9. Scale bar, $200 \mu \mathrm{m}$. (C) triMeH3K9 expression in pancreatic cancer and adjacent normal tissues was assessed semi-quantitatively by staining intensity. The difference between the two groups was analyzed by $\chi^{2}$ test. ${ }^{* *} \mathrm{P}<0.01$. (D) Overall survival according to the expression levels of triMeH3K9. triMeH3K9, histone H3 trimethyl K9.

the patients were numbered as aforementioned. The senescence score was the sum of the number generated from the expression levels of CDKN1A and Ki67. The larger summed number was associated with a more senescent state. Kaplan-Meier analysis and log-rank test were used to assess the association between the senescence score and survival. RNA-seq and sample profiling dataset of GSE140077 (19) was obtained from the Gene Expression Omnibus (GEO) database (https://www.ncbi. nlm.nih.gov/geo/) and used for comparison of gene expression levels between the BxPC-3 gemcitabine-resistant cell line and the parental cell line. For TCGA and GEO databases, the expression levels were transformed into $\log 2(\mathrm{TPM}+1)$ for subsequent analyses.

Statistical analysis. Normally distributed data were presented as the mean \pm SD of $\geq 3$ independent experiments. The unpaired Student's t-test was used to compare differences between two groups. Data were analyzed via one-way ANOVA followed by Dunnett's post-hoc test for multiple comparisons. Frequencies of categorical variables were compared using $\chi^{2}$ test. Statistical analyses were performed using SPSS 19.0 software (IBM Corp.). All statistical tests were two-tailed, and $\mathrm{P}<0.05$ was considered to indicate a statistically significant difference.

\section{Results}

Association between cellular senescence and prognosis in patients with pancreatic cancer. Cellular senescence is often observed in clinical pancreatic cancer tissues (20). Therefore, the present study investigated whether there was an association between cellular senescence and prognosis of pancreatic cancer. First, the clinical relevance of cellular senescence was assessed with classification based on CDKN1A/p21 positivity in combination with $\mathrm{Ki} 67$ negativity in pancreatic cancer samples from TCGA dataset. The results demonstrated that a higher senescence level tended to be associated with improved patient prognosis (Fig. 1A).

Considering that the formation of senescence-associated heterochromatic foci specifically enriched for $\mathrm{H} 3 \mathrm{~K} 9 \mathrm{me} 3$ has been implicated in cellular senescence (21), the present study further examined the expression levels of triMeH3K9 by immunohistochemistry in tissue microarrays containing 100 cases of pancreatic tumors and 80 cases of adjacent non-tumor tissues (Fig. 1B). It was observed that low triMeH3K9 expression (staining index, 0-3) was detected in 92 of the examined PDAC tumors versus 14 of the adjacent non-tumor tissues (Fig. 1C). However, when the 100 cases of PDAC tissues were divided into two groups, namely a positive and a negative triMeH3K9 

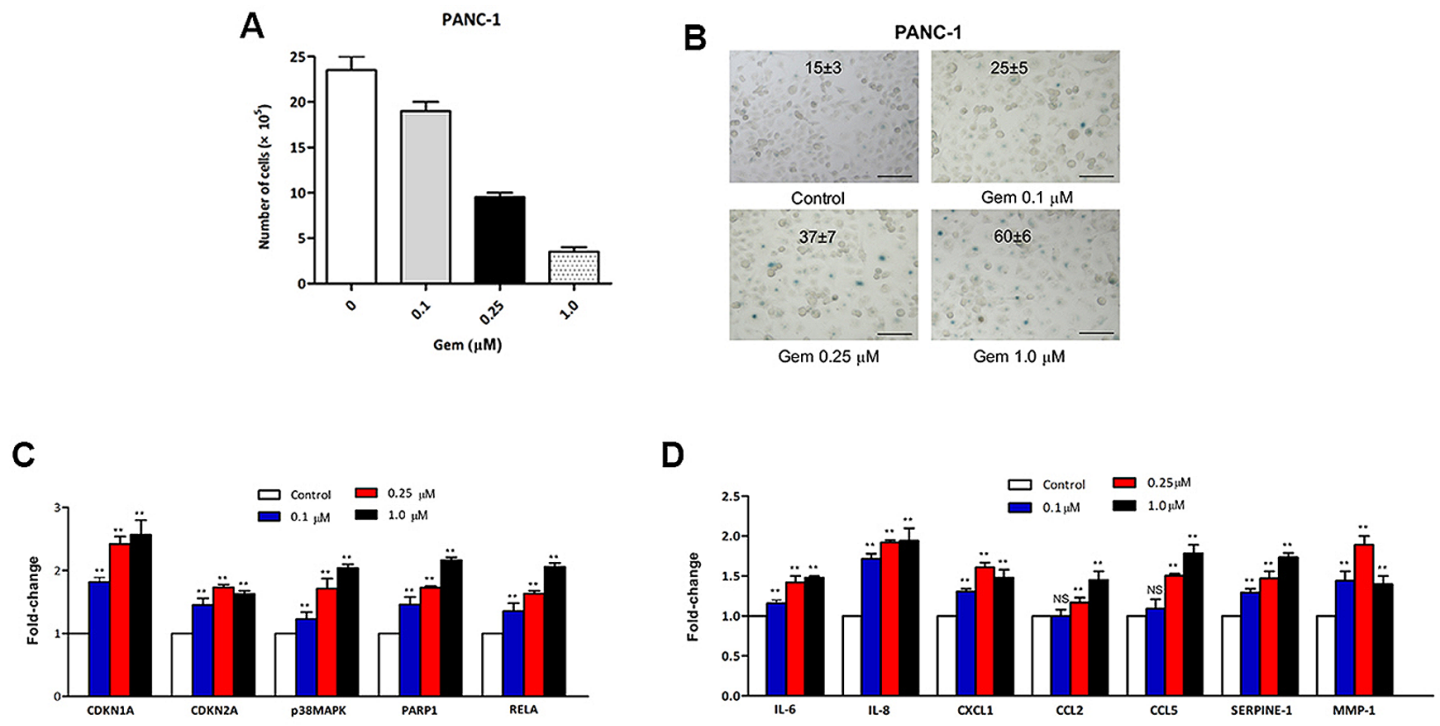
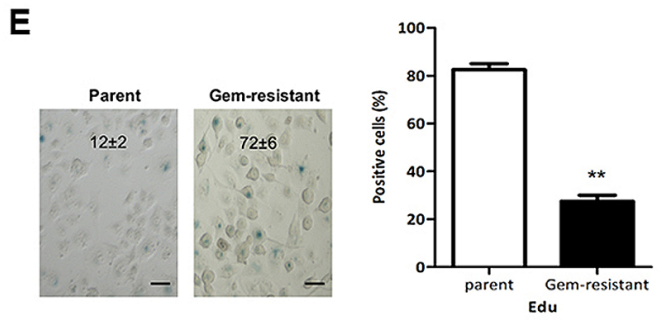

G

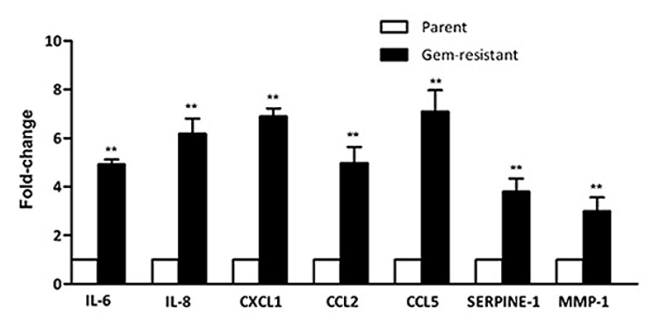

I

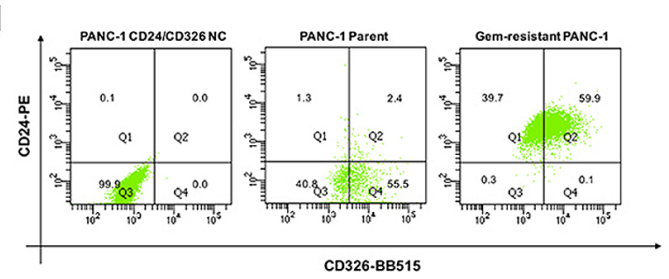

$\mathbf{F}$

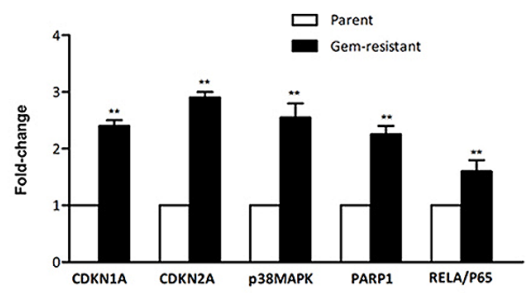

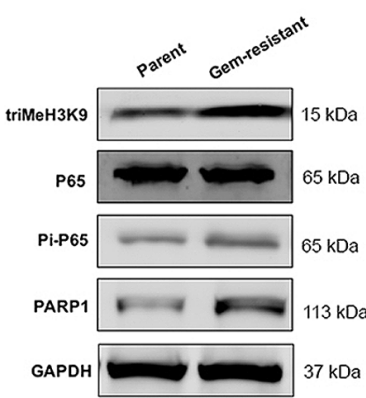

白 BXPC-3-GR 它 $8 \times P C-3$

$\mathrm{H}$

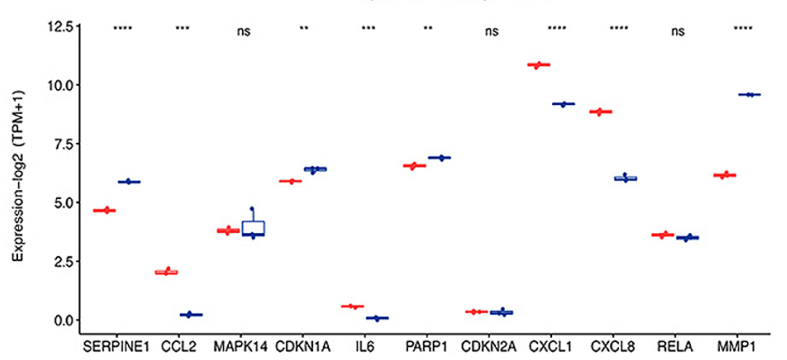

K

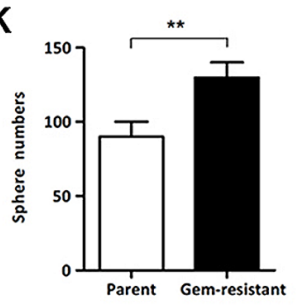

Figure 2. Gem-induced senescent pancreatic cancer cells possess phenotypic and functional stemness features. (A) PANC-1 cells were treated with the indicated doses of gem for $24 \mathrm{~h}$, and the cells were counted after 3 days. (B) PANC-1 cells exposed to gem were stained for SA- $\beta$-Gal activity. The values shown in the panels represent the percentage \pm SD of SA- $\beta$-Gal-positive cells. Scale bar, $100 \mu \mathrm{m}$. Cells were harvested and subjected to RT-qPCR to detect (C) senescence-associated signaling and (D) secretion of molecular mRNA. ${ }^{* *} \mathrm{P}<0.01$ vs. control. (E) Gem-resistant PANC-1 cells were either fixed and stained for SA- $\beta-$ Gal (left), or incubated for $24 \mathrm{~h}$ with EdU and then fixed and stained (right). The results are shown as the percentage of positive cells ( $>100$ cells scored; $\mathrm{n}=3$ independent experiments). ${ }^{* *} \mathrm{P}<0.01$ vs. parent. Scale bar, $50 \mu \mathrm{m}$. (F) Relative expression levels of senescence-associated signaling molecules detected by qPCR (left panel) and western blotting (right) panel in gem-resistant PANC-1 cells. ${ }^{*} \mathrm{P}<0.01$ vs. parent. (G) RT-qPCR analysis of mRNA levels encoding the senescence-associated secretory phenotype in gem-resistant PANC-1 cells. ${ }^{* *} \mathrm{P}<0.01$ vs. parent. (H) Expression profile of senescence-associated genes in gem-resistant and parental BxPC-3 cells, obtained from the GSE140077 dataset from the Gene Expression Omnibus database. ${ }^{* *} \mathrm{P}<0.01 ;{ }^{* * *} \mathrm{P}<0.001$; ${ }^{* * * * *} \mathrm{P}<0.0001$; ns, not significant. Ratio and the potential of pancreatic cancer stem cells were increased in gem-resistant PANC-1 cells. (I) CD24 ${ }^{+} \mathrm{CD} 326^{+}$ subpopulation, (J) ALDH ${ }^{+}$subpopulation and $(\mathrm{K})$ sphere numbers in parental and gem-resistant PANC-1 cells. ${ }^{* *} \mathrm{P}<0.01$. SA- $\beta$-Gal, senescence-associated $\beta$-galactosidase; RT-qPCR, reverse transcription-quantitative PCR; Gem, gemcitabine; triMeH3K9, histone H3 trimethyl K9; PARP1, poly (ADP-ribose) polymerase 1; CDKN, cyclin-dependent kinase inhibitor; NC, negative control; ALDH, aldehyde dehydrogenase. 
A
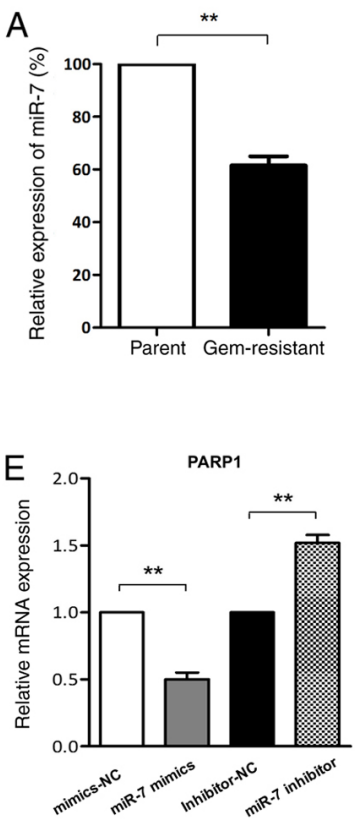

B

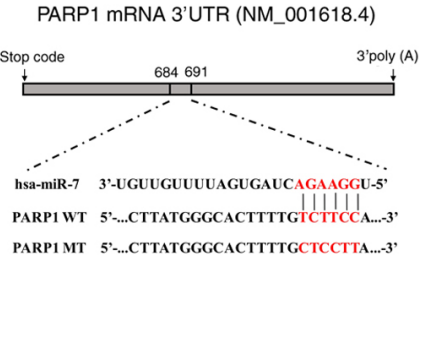

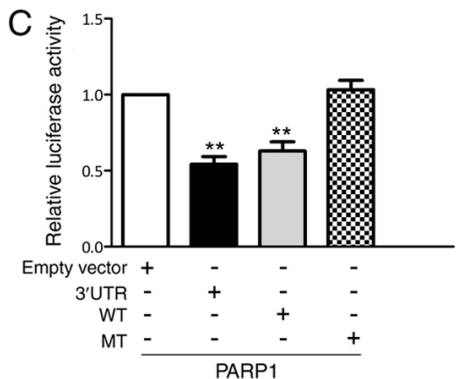

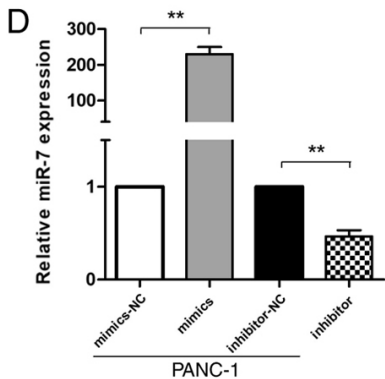

$\mathrm{F}$

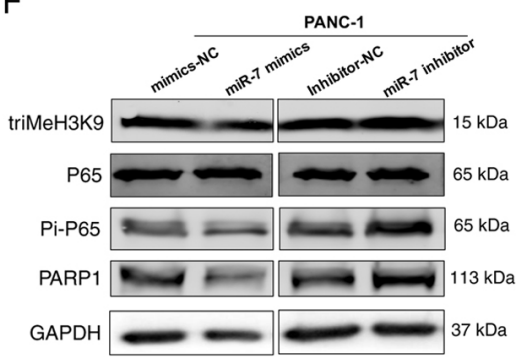

G

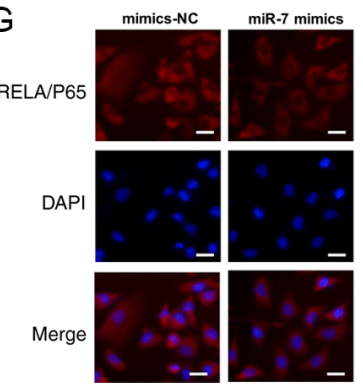

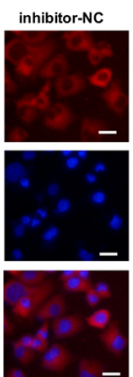

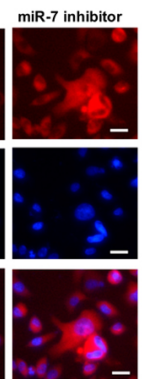

Figure 3. Gem-induced senescence is repressed by miR-7 via targeting PARP1/NF- $\mathrm{B}$ signaling in pancreatic cancer cells. (A) miR-7 expression by qPCR assay in parental and gem-resistant PANC-1 cells. ${ }^{* *} \mathrm{P}<0.01$. (B) Predicted miR-7-binding site sequence in the 3'-UTR of PARP1 and its WT or MT counterparts. (C) Luciferase reporter assays were performed at $48 \mathrm{~h}$ post-transfection to measure the relative luciferase activity on the corresponding constructs of 3'-UTR of PARP1 and their WT or MT counterparts for determination of PARP1 as miR-7 target in PANC-1 cells. ** $<0.01$ vs. empty vector. (D) Expression levels of miR-7 were analyzed by RT-qPCR in PANC-1 cells after transfection with miR-7 mimics or inhibitor. ${ }^{* *} \mathrm{P}<0.01$. (E) PARP1 mRNA expression in PANC-1 cells with overexpressed (mimics) or inhibited (inhibitor) miR-7 was detected by RT-qPCR. ${ }^{* *} \mathrm{P}<0.01$. (F) Western blot analysis of miR-7 mimics- or inhibitor-transfected PANC-1 cell lysates probed with antibodies against triMeH3K9, p65, Pi-p65 and PARP1. (G) Stimulation of NF- $\mathrm{B}$ activity in pancreatic cancer cells. Immunofluorescent staining with Pi-p65/RelA of PANC-1 cells transfected with miR-7 mimics or inhibitor. Nuclei were counterstained with DAPI. Scale bar, $50 \mu \mathrm{m}$. miR, microRNA; PARP1, poly (ADP-ribose) polymerase 1; WT, wild-type; MT, mutant; UTR, untranslated region; RT-qPCR, reverse transcription-quantitative PCR; Gem, gemcitabine; triMeH3K9, histone H3 trimethyl K9; NC, negative control; Pi, phospho.

expression group, it was observed that the average overall survival of the positive triMeH3K9 group was lower than that of the negative group (Fig. 1D). These results indicated that cellular senescence functioned as a potent tumor-suppressive process, as well as exerting deleterious effects in patients with pancreatic cancer.

Gemcitabine-induced senescent cells present novel stem-cell features in gemcitabine-resistant pancreatic cancer cells. The association between senescence and gemcitabine-resistance in pancreatic cancer was further explored. It is well known that gemcitabine is a first-line chemotherapeutic agent in pancreatic cancer. One anticancer activity of gemcitabine results from blocking DNA polymerase and causing DNA chain termination (22). As a consequence of DNA damage, senescence is activated in response to gemcitabine to prevent the propagation of pancreatic cancer cells (21). In the present study, PDAC PANC-1 cells were exposed to different doses of gemcitabine, and it was observed that gemcitabine induced senescence in PANC-1 cells, as measured by decreased cell proliferation (Fig. 2A), increased SA- $\beta$-Gal activity (Fig. 2B), elevated senescence-associated signaling (Fig. 2C) and increased expression levels of several SASP factors (Fig. 2D) mainly in a dose-dependent manner.

Furthermore, gemcitabine-resistant pancreatic cancer cells exhibited upregulated SA- $\beta$-Gal activity (Fig. 2E, left panel) and a significant decrease in DNA synthesis (Fig. 2E, right panel). In addition, senescence was confirmed by increased expression levels of CDKN1A/p21, CDKN2A/p16 and
p38MAPK/PARP1/NF- $\mathrm{B}$ senescent signaling, and of triMeH3K9 proteins by qPCR or western blot analysis (Fig. 2F), as well as elevated mRNA levels of SASP components (Fig. 2G) using qPCR. In addition, the transcriptional levels of senescence-associated genes in gemcitabine-resistant and parental BxPC-3 cells were determined by analyzing the GEO GSE140077 dataset. Notably, most key senescence-associated genes were significantly higher in gemcitabine-resistant BxPC-3 cells compared with their levels in parental BxPC-3 cells (Fig. 2H). These data indicated that gemcitabine induced senescence in pancreatic cancer cells, and that gemcitabine-resistant pancreatic cells had an increased senescence response.

It is known that cancer stem cells possess the ability to survive therapeutic intervention. Since a previous study reported that there was a cell-intrinsic association between the senescence program and the acquisition of self-renewing properties (23), the present study investigated whether the senescence condition promoted cancer stemness to induce resistance to gemcitabine in pancreatic cancer. Thus, stemness-associated membrane markers were analyzed in senescent PANC-1 cells with gemcitabine resistance. Acquisition of the stemness-associated markers CD24 and CD326 was observed in gemcitabine-resistant pancreatic cancer (Fig. 2I). In addition, as shown in Fig. 2J and $\mathrm{K}, \mathrm{ALDH}^{+}$subpopulations were markedly increased in gemcitabine-resistant pancreatic cancer, as revealed using the ALDEFLUOR ${ }^{\mathrm{TM}}$ assay, and the number of spheres of pancreatic cancer cells was significantly increased in the gemcitabine-resistant group compared with 
A

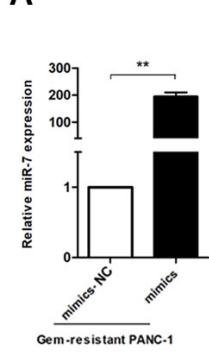

B

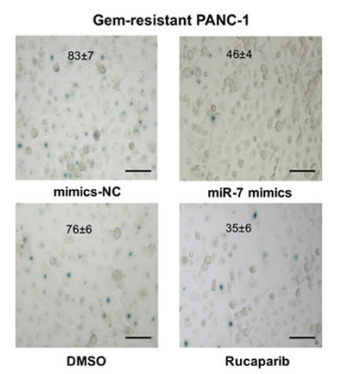

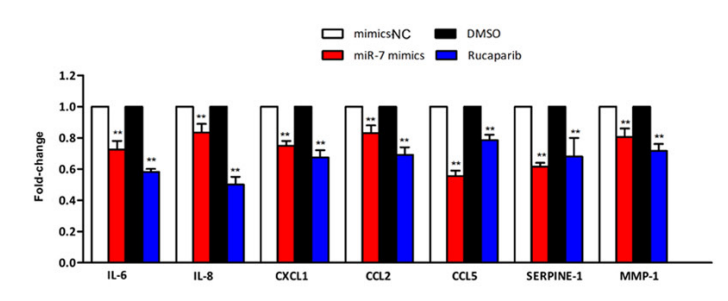

C

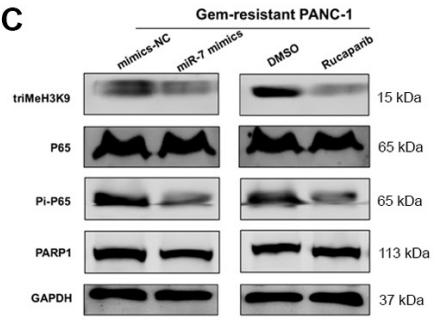

D

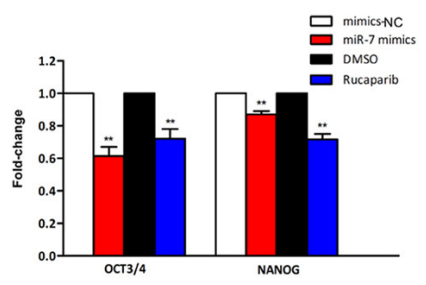

$\mathbf{E}$

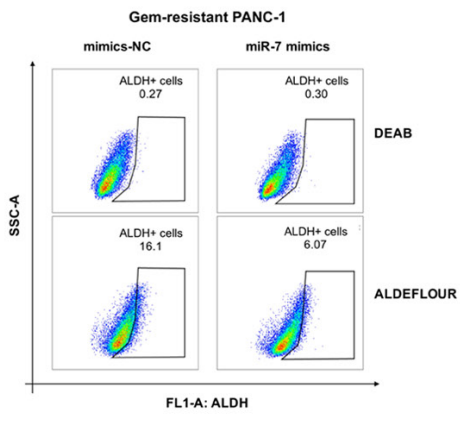

$\mathbf{F}$

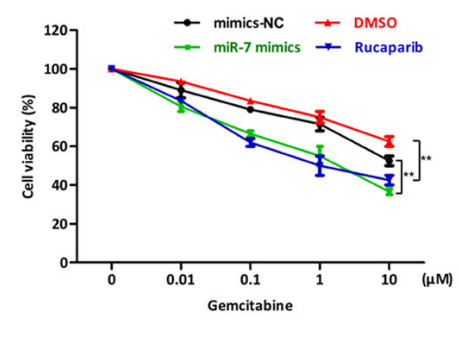

Figure 4. miR-7/PARP1/NF- $\kappa$ B axis is engaged in the chemo-sensitivity of PANC-1 cells to gemcitabine. (A) After being transfected with miR-7 mimics or treated with the PARP inhibitor rucaparib in gem-resistant PANC-1 cells, the relative miR-7 expression was detected by RT-qPCR (left; $\left.{ }^{* *} \mathrm{P}<0.01\right)$ and gem-resistant cells were subjected to senescence-associated $\beta$-galactosidase staining to determine the number of positive cells, which is shown as the mean percentage \pm SD (right). Scale bar, $100 \mu \mathrm{m}$. (B) mRNA transcripts of senescence-associated secretory phenotype and (C) protein levels of the PARP1/NF- $\kappa \mathrm{B}$ senescent axis were respectively analyzed by RT-qPCR and western blotting in gem-resistant PANC-1 cells transfected with miR-7 mimics or treated with rucaparib. ${ }^{* *} \mathrm{P}<0.01$ vs. mimics-NC or DMSO. (D) Expression levels of the indicated stem cell-associated genes in gem-resistant PANC-1 cells treated with miR-7 mimics or rucaparib was assessed by RT-qPCR. ${ }^{* *} \mathrm{P}<0.01$ vs. mimics-NC or DMSO. (E) ALDH activity with or without miR-7 mimics in gem-resistant PANC-1 cells was analyzed by flow cytometry. (F) Gem-resistant cancer cells were treated with gem for $24 \mathrm{~h}$ after being transfected with miR-7 mimics or incubated with rucaparib. The viability of the cells was determined by Cell Counting Kit- 8 assay. ${ }^{* *} \mathrm{P}<0.01$. RT-qPCR, reverse transcription-quantitative PCR; ALDH, aldehyde dehydrogenase; Gem, gemcitabine; triMeH3K9, histone H3 trimethyl K9; PARP1, poly (ADP-ribose) polymerase 1; miR, microRNA; NC, negative control.

that in the parental group. Hence, pancreatic cancer cells acquired novel stem-cell features upon chemotherapy-induced cellular senescence.

Gemcitabine-induced senescence is repressed by miR-7 via targeting PARPI/NF- $\kappa B$ signaling in pancreatic cancer cells. In our previous study, it was observed that miR-7 expression was decreased in pancreatic cancer, as well as in patients with gemcitabine-resistant pancreatic cancer, thus potentially being a biomarker for gemcitabine sensitivity in pancreatic cancer (24), and this may be associated with the regulation of senescence. The present study further explored whether miR-7 was involved in gemcitabine resistance via regulation of senescence in pancreatic cancer cells. Compared with that in the parental group, miR-7 exhibited a significantly decreased expression in the gemcitabine-resistant group of PANC-1 cells (Fig. 3A).

To further explore how miR-7 may be involved in gemcitabine resistance through the regulation of senescence, TargetScanHuman 7.2 was used, which identified PARP1 (GenBank NM_001618.4) as a potential target gene of miR-7. PARP1 is a genotoxic sensor involved in the activation of $N F-\kappa B$ and it mediates the pro-invasive capacity of senescence-associated secretome (25). Thus, the 3'-UTR of PARP1 (PPU), and the corresponding PPU-WT or PPU-MT sequence of the putative miR-7-binding site were cloned into the pmirGLO Dual-luciferase reporter vector (Fig. 3B). As a result, the luciferase activity in PANC-1 cells was significantly decreased after co-transfection of miR-7 mimics with pmirGLO-PPU or pmirGLO-PPU-WT for $48 \mathrm{~h}$, while the luciferase activity upon transfection with pmirGLO-PPU-MT was not affected by the inhibition of miR-7 mimics (Fig. 3C). Furthermore, when miR-7 was ectopically expressed (Fig. 3D), both the mRNA and protein levels of PARP1 were decreased using miR-7 mimics and increased using miR-7 inhibitors in PANC-1 cells (Fig. 3E and F).

Additionally, the effects of miR-7 on PARP1/NF- $\kappa$ B signaling were further evaluated. It is well known that $\mathrm{NF}-\kappa \mathrm{B}$ is present as an inactive cytoplasmic form, while activated $\mathrm{NF}-\kappa \mathrm{B}$ is translocated into the nucleus and binds to specific $\kappa \mathrm{B}$ enhancer elements in the promoter of targets genes. Thus, the present study investigated the expression levels of the p65/RelA subunit of NF- $\kappa \mathrm{B}$ by western blot analysis and immunofluorescence. The results revealed that, after inhibiting PARP1 expression using miR-7 mimics, the phosphorylation of p65/RelA decreased in PANC-1 cells (Fig. 3F). Additionally, under control conditions, p65/RelA localized mainly in the cytoplasm, while in the miR-7 inhibitor group, p65/RelA localized in the nucleus (Fig. 3G). A marked nuclear accumulation of p65/RelA in pancreatic cancer cells was observed upon miR-7 inhibitor treatment, which was stronger than that induced by miR-7 mimics (Fig. 3G). Furthermore, as expected, miR-7 negatively regulated triMeH3K9 formation (Fig. 3F). Overall, the current data demonstrated that PARP1 may be a 


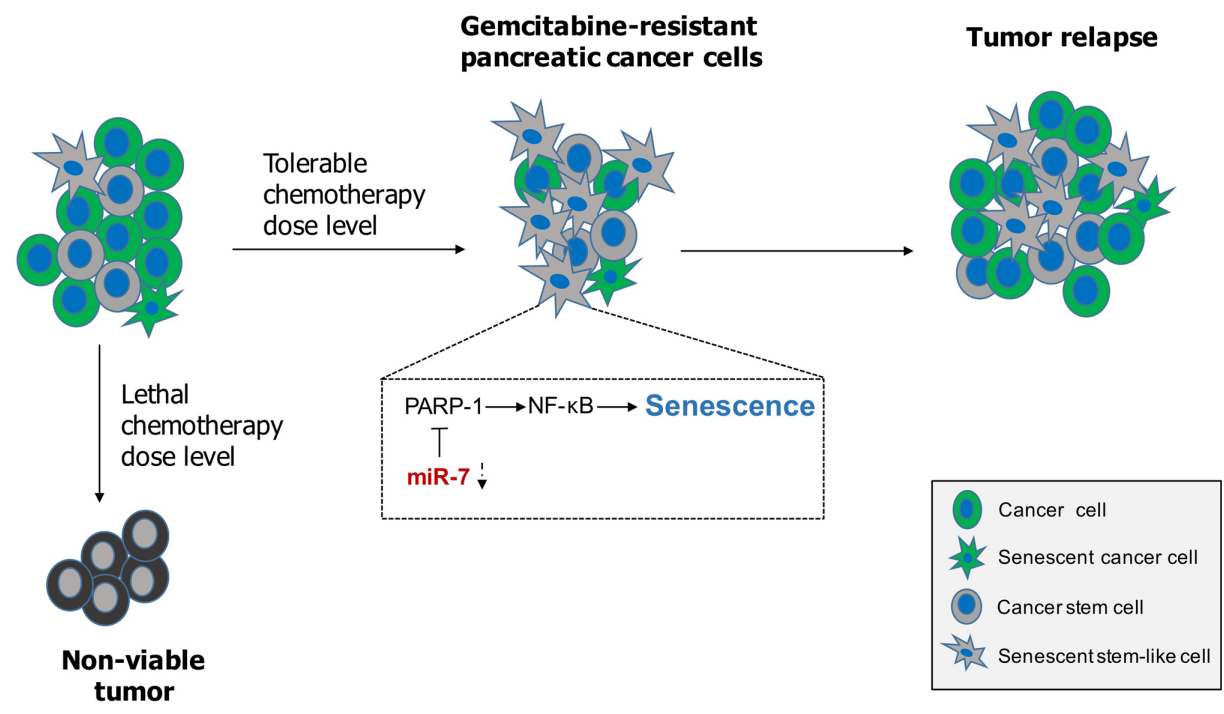

Figure 5. Schematic diagram of miR-7 modulating cellular senescence to relieve gemcitabine resistance via targeting PARP1/NF- $\mathrm{B}$ signaling in pancreatic cancer cells. Gemcitabine-resistant pancreatic cancer cells surviving chemotherapy become senescent cells with stem-like features, which leads to cancer relapse. Gemcitabine-induced senescence may be repressed by miR-7 targeting PARP1/NF-кB signaling. Thus, overexpression of miR-7 may sensitize pancreatic cancer cells to gemcitabine. PARP1, poly (ADP-ribose) polymerase 1; miR, microRNA.

target of miR-7, and that overexpression of $\mathrm{miR}-7$ resulted in a marked decrease in cellular PARP1 and subsequent NF- $\mathrm{B}$ inactivation, further repressing the senescence-associated phenotype.

miR-7 restores sensitivity to gemcitabine by restraining cellular senescence in pancreatic cancer cells. The present study demonstrated that gemcitabine-resistant pancreatic cancer cells had increased chemotherapy-induced senescence, and senescence-associated reprogramming promoted pancreatic cancer stemness. Additionally, it revealed that miR-7 expression was decreased in gemcitabine-resistant PANC-1 cells, and that miR-7 may be an important regulator of cellular senescence via targeting PARP1/NF- $\kappa \mathrm{B}$ signaling. Therefore, miR-7 expression was restored in gemcitabine-resistant PANC-1 cells to determine if this could sensitize pancreatic cancer cells to gemcitabine by repressing cellular senescence and decreasing the population of cancer stem cells.

Gemcitabine-resistant PANC-1 cells were transfected with miR-7 mimics or treated with the PARP inhibitor rucaparib. After $48 \mathrm{~h}$, it was observed that the overexpression of miR-7 in gemcitabine-resistant PANC-1 cells markedly decreased cellular senescence, and comparable results were also obtained in the rucaparib group, including decreased SA- $\beta$-Gal activity (Fig. 4A), decreased expression levels of SASP (Fig. 4B) and downregulation of PARP1/NF- $\kappa$ B senescent signaling (Fig. 4C). Additionally, assessment of the mRNA expression levels of the cancer stem cell transcription factors OCT3/4 and NANOG, which are involved in pluripotent cell maintenance and differentiation (26), in gemcitabine-resistant PANC-1 cells revealed that miR-7 mimics or rucaparib treatment decreased the expression levels of these molecules compared with those observed after gemcitabine treatment alone in gemcitabine-resistant PANC-1 cells (Fig. 4D). Similarly, following ALDEFLOUR ${ }^{\mathrm{TM}}$ staining, the population of $\mathrm{ALDH}^{+}$cells in the miR-7 mimics group was decreased by $\sim 62 \%$ compared with that in the miR-7 mimics-NC group (Fig. 4E). Next, to evaluate the role of miR-7 in the gemcitabine sensitivity of pancreatic cancer cells, gemcitabine-resistant cells were treated with gemcitabine for $24 \mathrm{~h}$ after being transfected with miR-7 mimics or being incubated with rucaparib, and cell viability was evaluated 3 days later by CCK- 8 assay. The results revealed that overexpression of miR-7 or treatment with the PARP inhibitor rucaparib significantly increased the sensitivity of resistant PANC-1 cells to gemcitabine (Fig. 4F). The current findings suggested that PARP1 depletion by miR-7 may attenuate senescence and decrease the stem cell population, which may enhance the sensitivity of PANC-1 cells to gemcitabine.

\section{Discussion}

Pancreatic cancer is an aggressive disease associated with major morbidity and mortality, and chemo-resistance is a major obstacle for PDAC treatment (27). In the last decade, gemcitabine has been considered as the standard treatment, and has been widely utilized as the first-line drug for advanced pancreatic cancer (3). However, development of chemo-resistance to gemcitabine severely limits the effectiveness of this chemotherapy (28). Therefore, it is urgent to clarify the underlying mechanism of the development of gemcitabine resistance.

Recent studies have demonstrated that cellular senescence may be involved in the acquisition of tumor cell resistance to chemotherapy (29). Cellular senescence is a state of permanent cell growth arrest that is often one of the terminal outcomes of chemotherapy (29). Traditionally, senescence induction is considered to be an important mechanism of cancer prevention and cellular aging (30). However, a recent study has revealed that senescence is a prominent solid tumor response to therapy in which cancer cells evade apoptosis and instead enter into a stable and prolonged cell cycle arrest (31). The present study detected TIS in a gemcitabine-resistant PDAC cell line 
through $\beta$-Gal activity assay, assessment of SASP expression and senescence signaling. As a result, it was demonstrated that senescence protected tumors from gemcitabine-induced cytotoxicity. Thus, the present study suggested that senescence may be involved in poor chemotherapy responses in pancreatic cancer.

Compared with tumors undergoing apoptosis, there are several problems with tumors becoming senescent. Firstly, senescence can send cancer cells into a state similar to extended sleep, in which cells are alive but not dividing; therefore, tumor growth is arrested and does not regress (32). Senescence helps cancer cells avoid death and turns them into cancer stem cells. Secondly, a cell-cycle-targeting chemotherapeutic agent such as gemcitabine (33) will lose efficiency in senescent cells. Thirdly, the senescence-evoked cell-intrinsic factors can reprogram cancer cells into a stem-like state (23), and the subpopulation of chemotherapy-induced senescent cancer cells have demonstrated the capability of escaping, thus leading to relapse (29). Lastly, senescent tumor cells are metabolically active, and can secrete cytokines and other paracrine factors, which feed and stimulate the growth of adjacent cells (34). Thus, senescence is viewed as a potentially important target in cancer therapy.

Recently, the ectopic expression of miRNAs in the regulation and induction of senescence has been investigated (13). Our previous study demonstrated that miR-7 served as a marker for gemcitabine sensitivity in pancreatic cancer (24). In the present study, miR-7 expression was downregulated in accelerated senescent gemcitabine-resistant pancreatic cancer cells, implying that decreased miR-7 expression may be an important cause of TIS in gemcitabine-resistant pancreatic cancer cells. To explore the potential mechanism underlying the involvement of miR-7 on senescent signaling in gemcitabine-resistant PDAC cells, bioinformatics analysis and dual-luciferase reporter assays were utilized to predict the gene targets of miR-7. The results revealed that miR-7 directly targeted PARP1 and suppressed PARP1/NF- $\mathrm{B}$ signaling in gemcitabine-resistant PDAC cells. PARP1, which detects DNA damage (such as that mediated by chemotherapeutics) and drives DNA repair, acts upstream of $N F-\kappa B$, while the PARP1/NF- $\kappa \mathrm{B}$ signaling cascade activated during senescence drives the formation of a secretome endowed with pro-tumoral and pro-metastatic properties (25). The current results revealed that miR-7-mediated suppression of cellular senescence in pancreatic cancer cells was attributed to the effect of chemotherapy by blockade of the PARP1/NF- $\kappa \mathrm{B}$ signaling cascade, which is consistent with our previous study that miR-7 enhances the sensitivity to gemcitabine chemotherapy (24). Next, it was demonstrated that the influence of miR-7 co-treatment with gemcitabine on the viability of gemcitabine-resistant pancreatic cancer cells affected the stemness phenotype and senescence of the cells. The present results strongly suggested that miR-7 exerted a regulatory effect on chemo-sensitivity via inhibition of senescence-like phenotypes in pancreatic cancer.

In conclusion, cellular senescence appears to be primarily a beneficial response by keeping tumor growth in check. However, activating a senescence program within tumor stem cells may exert potential detrimental effects on chemotherapy resistance (Fig. 5). In the present study, miR-7 significantly inhibited senescence-like changes to sensitize pancreatic cancer to gemcitabine through PARP1/NF- $\kappa \mathrm{B}$ signaling. Attenuating senescence via miR-7 may contribute to fight against gemcitabine resistance in pancreatic cancer in vitro. However, there are several limitations in the present study, including the use of only one pancreatic cancer cell line and performing only in vitro experiments. Thus, further research on more pancreatic cancer cell lines and in vivo studies are required to fully clarify the molecular mechanism of miR-7 and senescence in chemotherapy-resistant pancreatic cancer in the future.

\section{Acknowledgements}

Not applicable.

\section{Funding}

The present study was supported by funding from the National Natural Science Foundation of China (grant nos. 81802328 and 81773073), the Wenzhou Science and Technology Plan Project (grant nos. Y20180214 and Y20180846) and the Young Talents Program of the First Affiliated Hospital of Wenzhou Medical University (grant no. qnyc094).

\section{Availability of data and materials}

The datasets used and/or analyzed during the current study are available from the corresponding author on reasonable request, and the GSE140077 dataset is available in the Gene Expression Omnibus database (https://www.ncbi.nlm.nih.gov/ geo/query/acc.cgi?acc=GSE140077).

\section{Authors' contributions}

ZQY performed the experiments, analyzed the data and wrote the manuscript. HBC and TYZ performed the experiments and analyzed the data. ZC contributed to the conception, the design and the draft of the study. LT designed and supervised the study, interpreted the data and revised the manuscript. DNG designed and supervised the study, interpreted the data and wrote the manuscript. All authors read and approved the final manuscript.

\section{Ethics approval and consent to participate}

Not applicable.

\section{Patient consent for publication}

Not applicable.

\section{Competing interests}

The authors declare that they have no competing interests.

\section{References}

1. Siegel RL, Miller KD and Jemal A: Cancer statistics, 2019. CA Cancer J Clin 69: 7-34, 2019. 
2. Neoptolemos JP, Kleeff J, Michl P, Costello E, Greenhalf W and Palmer DH: Therapeutic developments in pancreatic cancer: Current and future perspectives. Nat Rev Gastroenterol Hepatol 15: 333-348, 2018.

3. Mohammed S, Van Buren G II and Fisher WE: Pancreatic cancer: Advances in treatment. World J Gastroenterol 20: 9354-9360, 2014.

4. Marino Gammazza A, Campanella C, Barone R, Caruso Bavisotto C, Gorska M, Wozniak M, Carini F, Cappello F, D'Anneo A, Lauricella M, et al: Doxorubicin anti-tumor mechanisms include Hsp60 post-translational modifications leading to the Hsp60/p53 complex dissociation and instauration of replicative senescence. Cancer Lett 385: 75-86, 2017.

5. Li W, Wang W, Dong H, Li Y, Li L, Han L, Han Z, Wang S, Ma D and Wang H: Cisplatin-induced senescence in ovarian cancer cells is mediated by GRP78. Oncol Rep 31: 2525-2534, 2014.

6. Lee JJ, Park IH, Rhee WJ, Kim HS and Shin JS: HMGB1 modulates the balance between senescence and apoptosis in response to genotoxic stress. FASEB J 33: 10942-10953, 2019.

7. Perez-Mancera PA, Young AR and Narita M: Inside and out: The activities of senescence in cancer. Nat Rev Cancer 14: 547-558, 2014.

8. Dorr JR, Yu Y, Milanovic M, Beuster G, Zasada C, Däbritz JH, Lisec J, Lenze D, Gerhardt A, Schleicher K, et al: Synthetic lethal metabolic targeting of cellular senescence in cancer therapy. Nature 501: 421-425, 2013.

9. Kaur A, Webster MR, Marchbank K, Behera R, Ndoye A, Kugel CH III, Dang VM, Appleton J, O'Connell MP, Cheng P, et al: sFRP2 in the aged microenvironment drives melanoma metastasis and therapy resistance. Nature 532: 250-254, 2016.

10. Sun Y, Coppe JP and Lam EW: Cellular senescence: The sough or the unwanted? Trends Mol Med 24: 871-885, 2018.

11. Song Y, Baba T and Mukaida N: Gemcitabine induces cell senescence in human pancreatic cancer cell lines. Biochem Biophys Res Commun 477: 515-519, 2016.

12. Hua YQ, Zhu YD, Xie GQ, Zhang K, Sheng J, Zhu ZF, Ning ZY, Chen H, Chen Z, Meng ZQ and Liu LM: Long non-coding SBF2-AS1 acting as a competing endogenous RNA to sponge microRNA-142-3p to participate in gemcitabine resistance in pancreatic cancer via upregulating TWF1. Aging (Albany NY) 11: 8860-8878, 2019.

13. Williams J, Smith F, Kumar S, Vijayan M and Reddy PH: Are microRNAs true sensors of ageing and cellular senescence? Ageing Res Rev 35: 350-363, 2017.

14. Gu DN, Jiang MJ, Mei Z, Dai JJ, Dai CY, Fang C, Huang Q and Tian L: microRNA-7 impairs autophagy-derived pools of glucose to suppress pancreatic cancer progression. Cancer Lett 400: 69-78, 2017.

15. Luo H, Liang H, Chen Y, Chen S, Xu Y, Xu L, Liu J, Zhou K, Peng J, Guo G, et al: miR-7-5p overexpression suppresses cell proliferation and promotes apoptosis through inhibiting the ability of DNA damage repair of PARP-1 and BRCA1 in TK6 cells exposed to hydroquinone. Chem Biol Interact 283: 84-90, 2018.

16. Lai J, Yang H, Zhu Y, Ruan M, Huang Y and Zhang Q MiR-7-5p-mediated downregulation of PARP1 impacts DNA homologous recombination repair and resistance to doxorubicin in small cell lung cancer. BMC Cancer 19: 602, 2019.

17. Livak KJ and Schmittgen TD: Analysis of relative gene expression data using real-time quantitative PCR and the 2(-Delta Delta C(T)) method. Methods 25: 402-408, 2001.

18. Li L, Hao X, Qin J, Tang W, He F, Smith A, Zhang M, Simeone DM, Qiao XT, Chen ZN, et al: Antibody against CD44s inhibits pancreatic tumor initiation and postradiation recurrence in mice. Gastroenterology 146: 1108-1118, 2014.
19. Zhou J, Zhang L, Zheng H, Ge W, Huang Y, Yan Y, Zhou X, Zhu W, Kong Y, Ding Y and Wang W: Identification of chemoresistance-related mRNAs based on gemcitabine-resistant pancreatic cancer cell lines. Cancer Med 9: 1115-1130, 2020.

20. Cheng Q, Ouyang X, Zhang R, Zhu L and Song X: Senescence-associated genes and non-coding RNAs function in pancreatic cancer progression. RNA Biol 17: 1693-1706, 2020.

21. Perez RF, Tejedor JR, Bayon GF, Fernández AF and Fraga MF: Distinct chromatin signatures of DNA hypomethylation in aging and cancer. Aging Cell 17: e12744, 2018.

22. Burris HA III, Moore MJ, Andersen J, Green MR, Rothenberg ML, Modiano MR, Cripps MC, Portenoy RK, Storniolo AM, Tarassoff P, et al: Improvements in survival and clinical benefit with gemcitabine as first-line therapy for patients with advanced pancreas cancer: A randomized trial. J Clin Oncol 15: 2403-2413, 1997.

23. Milanovic M, Fan DNY, Belenki D, Däbritz JHM, Zhao Z, Yu Y, Dörr JR, Dimitrova L, Lenze D, Monteiro Barbosa IA, et al: Senescence-associated reprogramming promotes cancer stemness. Nature 553: 96-100, 2018.

24. Ye ZQ, Zou CL, Chen HB, Jiang MJ, Mei Z and Gu DN: MicroRNA-7 as a potential biomarker for prognosis in pancreatic cancer. Dis Markers 2020: 2782101, 2020

25. Ohanna M, Giuliano S, Bonet C, Imbert V, Hofman V, Zangari J, Bille K, Robert C, Bressac-de Paillerets B, Hofman P, et al: Senescent cells develop a PARP-1 and nuclear factor- $\{$ kappa $\}$ B-associated secretome (PNAS). Genes Dev 25: 1245-1261, 2011.

26. Suresh B, Lee J, Kim KS and Ramakrishna S: The importance of ubiquitination and deubiquitination in cellular reprogramming. Stem Cells Int 2016: 6705927, 2016.

27. Dauer P, Nomura A, Saluja A and Banerjee S: Microenvironment in determining chemo-resistance in pancreatic cancer: Neighborhood matters. Pancreatology 17: 7-12, 2017.

28. Binenbaum Y, Na'ara S and Gil Z: Gemcitabine resistance in pancreatic ductal adenocarcinoma. Drug Resist Updat 23: 55-68, 2015.

29. Guillon J, Petit C, Toutain B, Guette C, Lelièvre E and Coqueret O: Chemotherapy-induced senescence, an adaptive mechanism driving resistance and tumor heterogeneity. Cell Cycle 18: 2385-2397, 2019.

30. Calcinotto A, Kohli J, Zagato E, Pellegrini L, Demaria M and Alimonti A: Cellular senescence: Aging, cancer, and injury. Physiol Rev 99: 1047-1078, 2019.

31. Demaria M, O'Leary MN, Chang J, Shao L, Liu S, Alimirah F, Koenig K, Le C, Mitin N, Deal AM, et al: Cellular senescence promotes adverse effects of chemotherapy and cancer relapse. Cancer Discov 7: 165-176, 2017.

32. Craske MG and Barlow DH: Nocturnal panic. J Nerv Ment Dis 177: 160-167, 1989.

33. Hamed SS, Straubinger RM and Jusko WJ: Pharmacodynamic modeling of cell cycle and apoptotic effects of gemcitabine on pancreatic adenocarcinoma cells. Cancer Chemother Pharmacol 72: 553-563, 2013.

34. Acosta JC, Banito A, Wuestefeld T, Georgilis A, Janich P, Morton JP, Athineos D, Kang TW, Lasitschka F, Andrulis M, et al: A complex secretory program orchestrated by the inflammasome controls paracrine senescence. Nat Cell Biol 15: 978-990, 2013.

c) (7) $\Theta$ This work is licensed under a Creative Commons Attribution-NonCommercial-NoDerivatives 4.0 International (CC BY-NC-ND 4.0) License. 